\title{
The post-common-envelope binary central star of the planetary nebula ETHOS 1
}

\author{
James Munday, ${ }^{1,2 \star}$ David Jones, ${ }^{2,3} \dagger$ Jorge García-Rojas, ${ }^{2,3}$ Henri M.J. Boffin, ${ }^{4}$ \\ Brent Miszalski, ${ }^{5}$ Romano L.M. Corradi, ${ }^{6,2}$ Pablo Rodríguez-Gil, ${ }^{2,3}$ \\ María del Mar Rubio-Díez, ${ }^{7}$ Miguel Santander-García ${ }^{8}$ and Paulina Sowicka9 \\ ${ }^{1}$ Astrophysics Research Group, Faculty of Engineering and Physical Sciences, University of Surrey, Guildford, Surrey, GU2 7XH, United Kingdom \\ ${ }^{2}$ Instituto de Astrofísica de Canarias, E-38205 La Laguna, Spain \\ ${ }^{3}$ Departamento de Astrofísica, Universidad de La Laguna, E-38206 La Laguna, Spain \\ ${ }^{4}$ European Southern Observatory, Karl-Schwarzschild-str. 2, D-85748 Garching, Germany \\ ${ }^{5}$ Australian Astronomical Optics - Macquarie, Faculty of Science and Engineering, Macquarie University, North Ryde, NSW 2113, Australia \\ ${ }^{6}$ GRANTECAN, Cuesta de San José s/n, E-38712, Breña Baja, La Palma, Spain \\ ${ }^{7}$ Centro de Astrobiología (CSIC/INTA), 28850 Torrejón de Ardoz, Madrid, Spain \\ ${ }^{8}$ Observatorio Astronómico Nacional (OAN-IGN), Alfonso XII, 3, 28014, Madrid, Spain \\ ${ }^{9}$ Nicolaus Copernicus Astronomical Center, Bartycka 18, PL-00-716 Warsaw, Poland
}

Accepted 2020 September 07. Received 2020 September 07; in original form 2020 August 06

\begin{abstract}
We present a detailed study of the binary central star of the planetary nebula ETHOS 1 (PN G068.1+11.0). Simultaneous modelling of light and radial velocity curves reveals the binary to comprise a hot and massive pre-white-dwarf with an M-type main-sequence companion. A good fit to the observations was found with a companion that follows expected mass-temperature-radius relationships for low-mass stars, indicating that despite being highly irradiated it is consistent with not being significantly hotter or larger than a typical star of the same mass. Previous modelling indicated that ETHOS 1 may comprise the first case where the orbital plane of the central binary does not lie perpendicular to the nebular symmetry axis, at odds with the expectation that the common envelope is ejected in the orbital plane. We find no evidence for such a discrepancy, deriving a binary inclination in agreement with that of the nebula as determined by spatio-kinematic modelling. This makes ETHOS 1 the ninth post-common-envelope planetary nebula in which the binary orbital and nebular symmetry axes have been shown to be aligned, with as yet no known counter-examples. The probability of finding such a correlation by chance is now less than $0.00002 \%$.
\end{abstract}

Key words: binaries: close - planetary nebulae: individual: PN G068.1+11.0 - white dwarfs - stars: AGB and post-AGB

\section{INTRODUCTION}

A significant fraction (perhaps even a majority; De Marco et al. 2015; Douchin et al. 2015) of planetary nebulae (PNe) are the product of interactions in close binary stars (Miszalski et al. 2009). However, despite their clear importance in understanding PNe (Jones \& Boffin 2017; Boffin \& Jones 2019), the nature of those binary interactions and, in particular, the common envelope (CE) phase is still rather poorly understood. A key step towards furthering our understanding of these systems is to observationally constrain their parameters such that they can be used to confront models. In recent years, much progess has been made with a number of post-CE

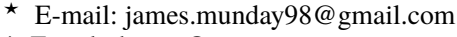

$\dagger$ Email: djones@iac.es central stars having their masses, radii and temperatures derived via simultaneous modelling of light- and radial velocity curves (see e.g.; Jones et al. 2015; Hillwig et al. 2016, 2017; Jones et al. 2019, 2020a), however for the vast majority of the sixty or so known post-CE central stars only their orbital periods are known.

ETHOS $1 \quad$ (PN G068.1+11.0, $\quad \alpha=19^{h} 16^{m} 31.5^{s}$, $\delta=-36^{\circ} 09^{\prime} 48^{\prime \prime}$ ) was found by Miszalski et al. (2011) to host a photometrically-variable central star with a period of roughly 0.535 days. Spectroscopy of the central star revealed the presence of a complex of emission lines typical of irradiation of a main sequence companion by a hot central star, strongly supporting a binary hypothesis for the photometric variability. The observed amplitude of this variability ( $\sim 0.8 \mathrm{mag}$ in the $i$-band), makes that of the central star of ETHOS 1 one of the most extreme irradiation effects known amongst the central stars of planetary nebulae 
(CSPNe). Furthermore, the nebula itself displays a bipolar central structure with extended jets which are found to pre-date the central regions (as has been found in other post-CE PNe; Mitchell et al. 2007; Boffin et al. 2012). This combination of extreme irradiation and bipolar jets draws clear parallels with another post-CE CSPN - that of the Necklace nebula (Corradi et al. 2011), the companion of which has been shown to be a carbon dwarf having been polluted with carbon-enriched material from the nebular progenitor (Miszalski et al. 2013). Previous light- and radial-velocity curve modelling efforts by Mitrofanova et al. (2016) derived an orbital inclination at odds with the nebular inclination (Miszalski et al. 2011) - indicating that ETHOS 1 could be the first post-CE PN not to follow the theoretically expected correlation between the two, which is a natural consequence of the $\mathrm{CE}$ being ejected preferentially in the orbital plane (Hillwig et al. 2016).

In this paper, we present a detailed modelling of the post-CE CSPN of ETHOS 1, based on newly acquired spectroscopy and photometry (combined with the photometry presented in Miszalski et al. 2011), in order to constrain the binary parameters - including the orbital inclination and thus probe the possible discrepancy between the binary and nebular inclinations. Section 2 describes the observations and data reduction, while in Section 3 we present the simultaneous light- and radial velocity curve modelling (and comparison to models published in the literature), before concluding in Section 4.

\section{OBSERVATIONS}

\subsection{Photometry}

Time-series photometry of ETHOS 1 in the $i$-band, acquired using the MEROPE camera (Davignon et al. 2004) mounted on the 1.2-m Flemish Mercator Telescope between August 24 and September 4 2009, was already presented in Miszalski et al. (2011). The same data is reanalysed here in order to provide a longer time baseline with which to constrain the orbital period as well as model the photometric variability.

Further observations were obtained using the Wide Field Camera (WFC) of the 2.5-m Isaac Newton Telescope (INT) on the nights of August 21, 22 and 23, as well as November 1 2015, and with the IO:O instrument of the 2-m Liverpool Telescope (LT; Steele et al. 2004) on multiple nights between April 29 and July 1 2020. With both instruments, exposures were acquired through $B, g, r$ and $i$ filters with integration times of $120 \mathrm{~s}, 90 \mathrm{~s}, 120 \mathrm{~s}$, $90 \mathrm{~s}$ respectively. For the exact dates of all exposures, we refer the reader to the online data or the relevant online data archives.

The Mercator and INT observations were debiased and flatfielded using standard routines of the astropy-affiliated python package ccdproc (Craig et al. 2017), while those from the LT were reduced with the IO:O pipeline. Differential photometry of the central stars was then performed against field stars using the SEP implementation of the SExTRACTOR algorithms (Bertin \& Arnouts 1996; Barbary 2016), before being placed on an apparent magnitude scale using observations of standard stars taken during the course of the observations. The resulting photometric measurements are available in the online data.

The $i$-band light curve (which offers the longest time span of observations) was searched for periodicities using the PERIOD package of the sTARLINK software suite (Dhillon et al. 2001; Currie et al. 2008). The determined ephemeris is

$\mathrm{HJD}_{\min }=2455076.0352(4)+0.5351263(2) E$ for the Heliocentric Julian Date of the photometric minimum $\left(\mathrm{HJD}_{\min }\right)$. The refined period derived here is entirely compatible with that found by Miszalski et al. (2011), however the new, more precise LT and INT data allow us to derive a more accurate timing for the photometric minimum (which is roughly $5 \sigma$ from the value found by Miszalski et al. 2011). The phase-folded light curves for each filter are shown in figure 1.

The folded light curves all display roughly sinusoidal variability with amplitude increasing with the effective wavelength of the filter (from semi-amplitude $\sim 0.7 \mathrm{mag}$ in $B$ to $\sim 0.8 \mathrm{mag}$ in $i$, well aligned with the amplitudes found by Mitrofanova et al. 2016). As highlighted by Miszalski et al. (2011), the large amplitude of variability places the central star of ETHOS 1 among a small group of very extreme irradiation effect binaries (Miszalski et al. 2009; Exter et al. 2003; Corradi et al. 2011), all of which are found inside PNe. While the amplitude of variability is found to increase with effective wavelength of the filter, the brightness at minimum is found to decrease (reddened $B-r \approx-0.35$ and $r-i \approx 0.19$ ). This behaviour is generally consistent with the presence of a very hot central star irradiating a main-sequence companion.

\subsection{Spectroscopy}

The central star system of ETHOS 1 was observed using the bluearm of the Dual Imaging Spectrograph (DIS) mounted on the $3.5 \mathrm{~m}$ Astrophysical Research Consortium (ARC) telescope at the Apache Peak Observatory on the nights of June 21, 23 and 242012 (for exact times, please see table 1). A 1.5" wide longslit was employed in conjunction with the B1200 grating to provide a resolution of $\sim 1.8 \AA$ between $3900-5050 \AA$. All exposures were of 1800-s duration and taken at a position angle of $149^{\circ}$ (along the jet axis). The data were debiased, flat-field corrected, optimally extracted and wavelength calibrated using standard IRAF routines ${ }^{1}$.

The central star system of ETHOS 1 was also observed using the blue-arm of the Intermediate-dispersion Spectrograph and Imaging System (ISIS) mounted on the 4.2-m William Herschel Telescope (WHT) on the nights of August 7 2015, September 2 and 32016 (for exact times, please see table 1). A 1" wide longslit was used along with the R1200B grating to provide a resolution of $\sim 0.8 \AA$ over the range of roughly $4250-5050 \AA$. All exposures were of 1800 -s duration and taken at the parallactic angle. The data were debiased and flat-fielded using day-time calibrations and wavelength calibration against $\mathrm{CuNe}+\mathrm{CuAr}$ arc lamp frames (taken either immediately before or after each observation). The data were then sky-subtracted and optimally extracted (following the algorithm of Horne 1986) to produce 1-D spectra of the central star, all using standard STARLINK routines (Shortridge et al. 2004; Currie et al. 2008).

Both the WHT-ISIS spectra and ARC-DIS spectra were then continuum subtracted before cross-correlation against a template comprising a flat continuum with the complex of irradiated emission lines (N III $\lambda 4634.14+4640.64 \AA$, C III $\lambda 4647.42+4650.25+4651.47 \AA$ and $\mathrm{C}$ IV $\lambda 4658.30 \AA$ as identified in figure 2) superimposed (as in Jones et al. 2020a). Unfortunately, just as in Miszalski et al. (2011), it was not possible to adequately

1 IRAF is distributed by the National Optical Astronomy Observatories, which are operated by the Association of Universities for Research in Astronomy, Inc., under cooperative agreement with the National Science Foundation. 

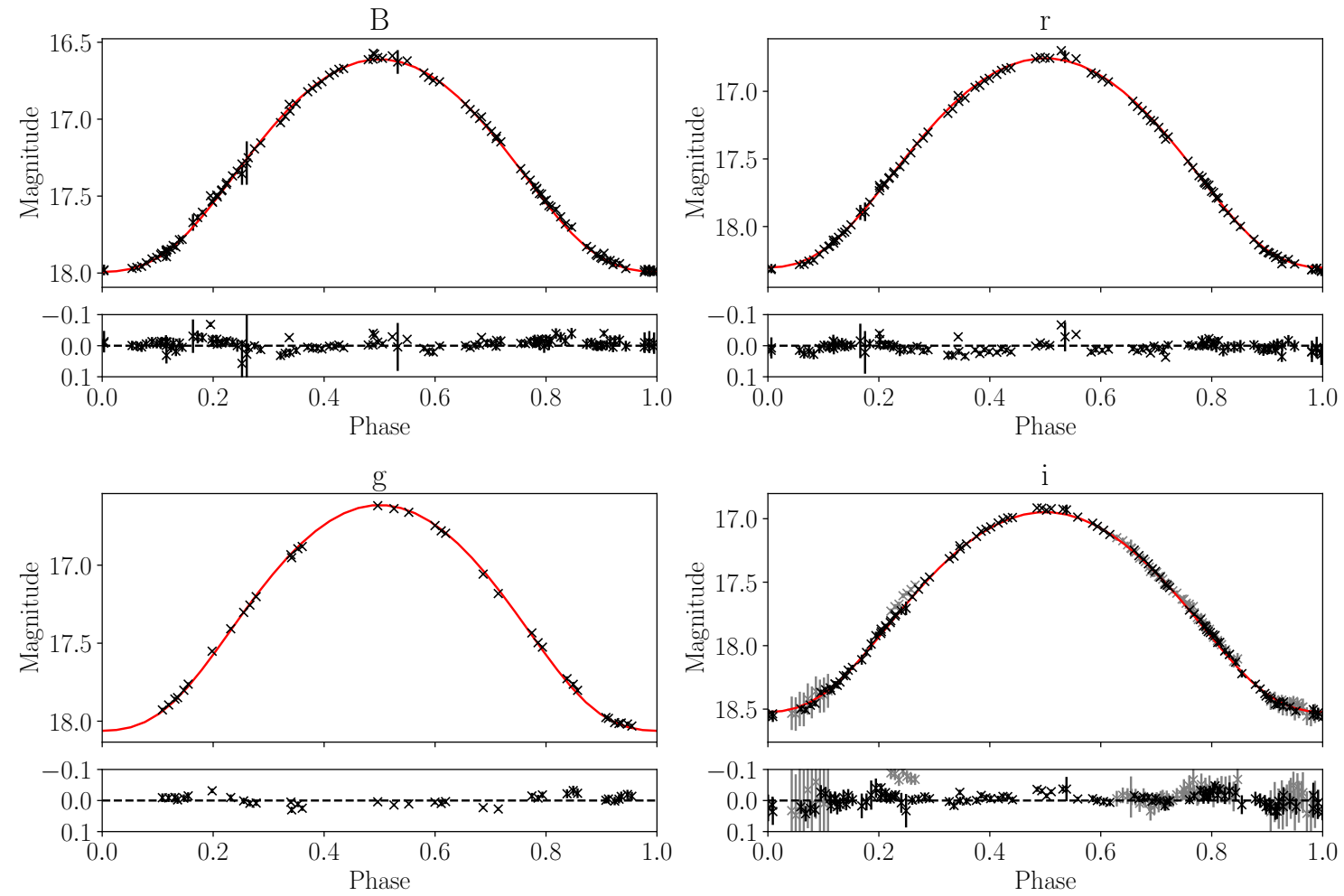

Figure 1. Phase-folded multi-band photometry of the central star system of ETHOS 1 overlaid on the best-fitting PHOEBE2 model light curves. The MercatorMerope $i$-band data, originally presented in Miszalski et al. (2011), is shown underlaid in grey beneath the more precise LT-IO:O and INT-WFC data. Beneath each light curve are the residuals between the best-fitting model and the data.

subtract the bright nebular emission in order to derive the radial velocities (RVs) of the hot component of the binary.

The RV measurements of the irradiated emission line complex, following heliocentric correction, are shown in table 1 while the data are shown folded on the ephemeris determined from the photometry in Figure 3. Upon inspection, the radial velocities present with a roughly sinusoidal variation with amplitude $\sim 125 \mathrm{~km} \mathrm{~s}^{-1}$ and a systemic velocity $\sim-10 \mathrm{~km} \mathrm{~s}^{-1}$. This amplitude is in good agreement with that determined by Mitrofanova et al. $(2016)^{2}$, however the systemic velocity is rather different. Mitrofanova et al. (2016, based on their figure 2) find a systemic velocity of approximately $105 \mathrm{~km} \mathrm{~s}^{-1}$, while the systemic velocity found here is much closer to that derived for the nebula, $v_{\text {sys }} \sim-20 \mathrm{~km} \mathrm{~s}^{-1}$, by Miszalski et al. (2011). It is also interesting to note that the systemic velocity implied by our measurements and those of the nebula are roughly consistent with Galactic rotation (accounting for a correction to the Local Standard of Rest), while the velocity of Mitrofanova et al. (2016) would likely indicate some form of runaway star.

2 Note that to derive the RV amplitude, Mitrofanova et al. (2016) claim to discard all measurements between phases $0.79-1.25$ based on the reduced strength of the emission-line complex around photometric minimum. However, it seems clear from their figure 4 c.f. their table 1 and our Figure 3, that they also discard at least two data points outside

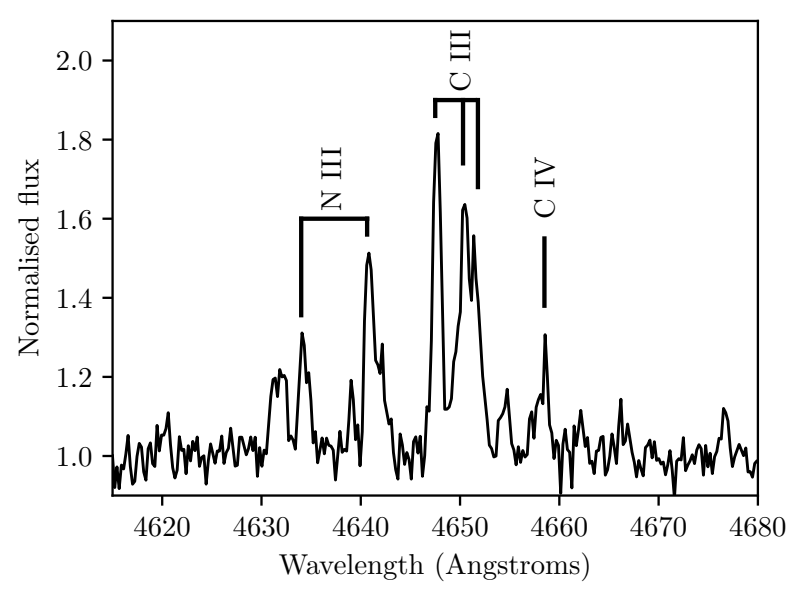

Figure 2. Normalised spectrum of the central star of ETHOS 1 (taken at phase $\phi=0.34$ ) showing the irradiated emission line complex used to derive the radial velocities of the companion. 
Table 1. Heliocentric radial velocity measurements of the irradiated emission line complex in the spectrum of the central star of ETHOS 1. The entry marked with an asterisk corresponds to the single measurement made by Miszalski et al. (2011) based on their VLT-FORS2 spectrum.

\begin{tabular}{rrrl}
\hline HJD & Phase & \multicolumn{2}{c}{ RV $\left(\mathrm{km} \mathrm{s}^{-1}\right)$} \\
\hline$* 2455026.69807$ & 0.80 & -122.0 & \pm 4.6 \\
2456099.89722 & 0.31 & 91.1 & \pm 15.0 \\
2456099.92182 & 0.36 & 84.9 & \pm 25.7 \\
2456101.90866 & 0.07 & 59.9 & \pm 33.5 \\
2456101.92978 & 0.11 & 61.2 & \pm 24.4 \\
2456103.89864 & 0.79 & -104.2 & \pm 43.5 \\
2457242.40598 & 0.34 & 111.5 & \pm 10.3 \\
2457242.42795 & 0.38 & 82.9 & \pm 13.3 \\
2457242.45300 & 0.42 & 63.8 & \pm 14.8 \\
2457242.47305 & 0.46 & 43.5 & \pm 34.0 \\
2457634.39103 & 0.85 & -97.7 & \pm 8.4 \\
2457634.41556 & 0.89 & -70.5 & \pm 23.7 \\
2457634.52229 & 0.09 & 31.9 & \pm 13.0 \\
2457634.54623 & 0.13 & 69.8 & \pm 11.7 \\
2457634.56991 & 0.18 & 98.9 & \pm 29.4 \\
2457634.59331 & 0.22 & 106.7 & \pm 28.0 \\
2457635.37578 & 0.69 & -127.1 & \pm 5.9 \\
2457635.39946 & 0.73 & -131.2 & \pm 22.0 \\
2457635.42283 & 0.77 & -130.1 & \pm 24.2 \\
2457635.47723 & 0.88 & -92.3 & \pm 6.2 \\
2457635.50076 & 0.92 & -64.9 & \pm 25.6 \\
\hline
\end{tabular}

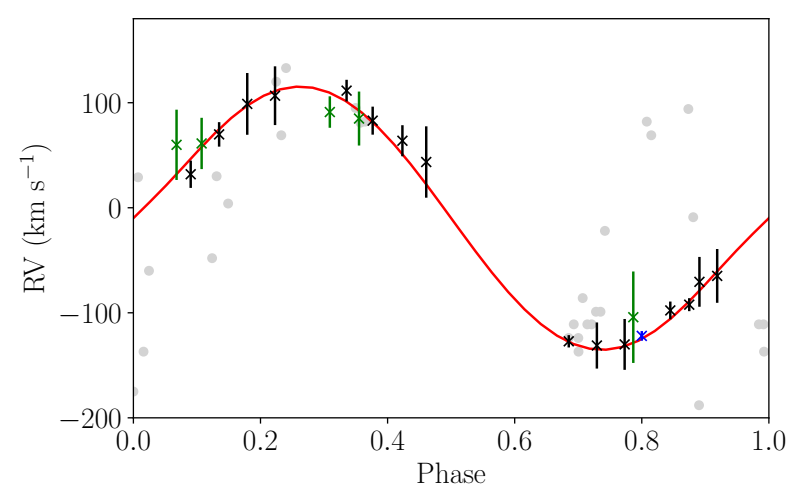

Figure 3. Phase-folded RVs of the irradiated emission line complex from the central star of ETHOS 1 (WHT-ISIS in black, ARC-DIS in green, and the single VLT-FORS2 point in blue taken from Miszalski et al. 2011), overlaid on the best-fitting PHOEBE2 model RV curve. The radial velocity measurements of Mitrofanova et al. (2016), offset by $-105 \mathrm{~km} \mathrm{~s}^{-1}$ and folded on the ephemeris determined in Section 2, are shown as grey circles (note that they do not quote any uncertainties).

\section{PHOEBE MODELLING}

The extracted light and radial velocity curves were modelled using the version 2.2 release of the PHOEBE2 code (Prša et al. 2016; Horvat et al. 2018; Jones et al. 2020b). Fitting was performed via a Markov Chain Monte Carlo (MCMC) method in python, using the EMCEE package, and executed on the LaPalma3 supercomputer (using the methodology presented in Jones et al. 2019).

The primary's temperature, mass and radius were allowed to vary freely, with limb-darkening coefficients extrapolated from the tables of Claret et al. (2020). PHOEBE2's interpolated limb- darkening, based on Castelli \& Kurucz (2003) atmospheres, were used for the secondary, while its albedo was allowed to vary freely in the physically-acceptable range 0.6-1.0 (Claret 2001). As there are minimal observational constraints for the secondary's mass (i.e. no radial velocities from the primary component) and radius (the system is not eclipsing), the mass, temperature and radius of the secondary were reduced to a single free parameter (the mass) with the others being set using the mass-temperature-radius relationship (MTRR) found for main-sequence stars by Eker et al. (2018, note that there is a typographic error in their mass-radius relationship for the low mass range ${ }^{3}$ which was discovered by our referee, Prof. Todd Hillwig). The final free parameters in the model are the binary inclination, allowed to vary freely over a range $\left(35^{\circ}<i<70^{\circ}\right)$ which covers both the solutions of Mitrofanova et al. $\left(2016,37^{\circ} \pm 1^{\circ}\right.$ and $\left.39^{\circ} \pm 0.2^{\circ}\right)$ and the inclination expected from spatio-kinematical modelling of the nebula (Miszalski et al. 2011, $60^{\circ} \pm 5^{\circ}$ ), and the systemic velocity (allowed to vary over the range, $\gamma=[-30$, $+10] \mathrm{km} \mathrm{s}^{-1}$ ).

The best-fitting model parameters (as derived from sampling the MCMC posteriors as shown in Figure 4) are listed in Table 2, while the corresponding model light- and RV-curves are shown underlaid on the data in Figures 1 and 3, respectively.

The model fits the observed spectroscopy and photometry well, with residuals generally being of the order of one uncertainty. The majority of photometric points where this is not the case, are likely a consequence of underestimated uncertainties as, while every attempt has been made to remove the nebular contamination, this is not reflected in the purely statistical uncertainties. The model residuals are, however, systematically larger than the uncertainties around the $i$-band minimum, perhaps indicative that the temperature of the secondary is over-estimated by the model (this is the only band where the companion will contribute significantly around photometric minimum due to the high temperature and luminosity of the hot component). Relaxing the MTRR could potentially resolve this discrepancy, but given the lack of additional constraints and the intrinsic problems involved in modelling irradiated binaries (see section 7 of Horvat et al. 2019, for a more detailed discussion), we feel that the forced-MTRR fit is reasonable. Similarly, the choice of model atmosphere for the secondary (Castelli \& Kurucz 2003, as is standard in PHOEBE2) may also be important with the derived secondary temperature lying so close to the lower boundary of the range covered by those atmospheres (the shape of the MCMC posteriors for $M_{2}$ in Figure 4 give some indication that this lower limit is hit by a number of chains). In spite of this, we still favour these atmospheres over the other options: PHOENIx (the range of which does not extend to high enough temperatures to model such extreme irradiation Husser et al. 2013) or black body atmospheres (which are a rather poor representation of the observed spectral energy distribution of low-mass dwarfs). It is important to note that forcing a lower inclination, similar to that found by Mitrofanova et al. (2016), does not improve the fit, instead leading to a larger discrepancy around the $i$-band minimum.

\subsection{Discrepancies with the modelling of Mitrofanova et al. (2016)}

Mitrofanova et al. (2016) presented a model of the CSPN of

3 The correct mass-radius relationship for the range $0.179 \leq \mathrm{M} / \mathrm{M}_{\odot} \leq 1.5$, as kindly provided by Prof. Z. Eker, should be: $\mathrm{R}=0.438(098) \times \mathrm{M}^{2}+0.479(180) \times \mathrm{M}+0.137(075)$. 


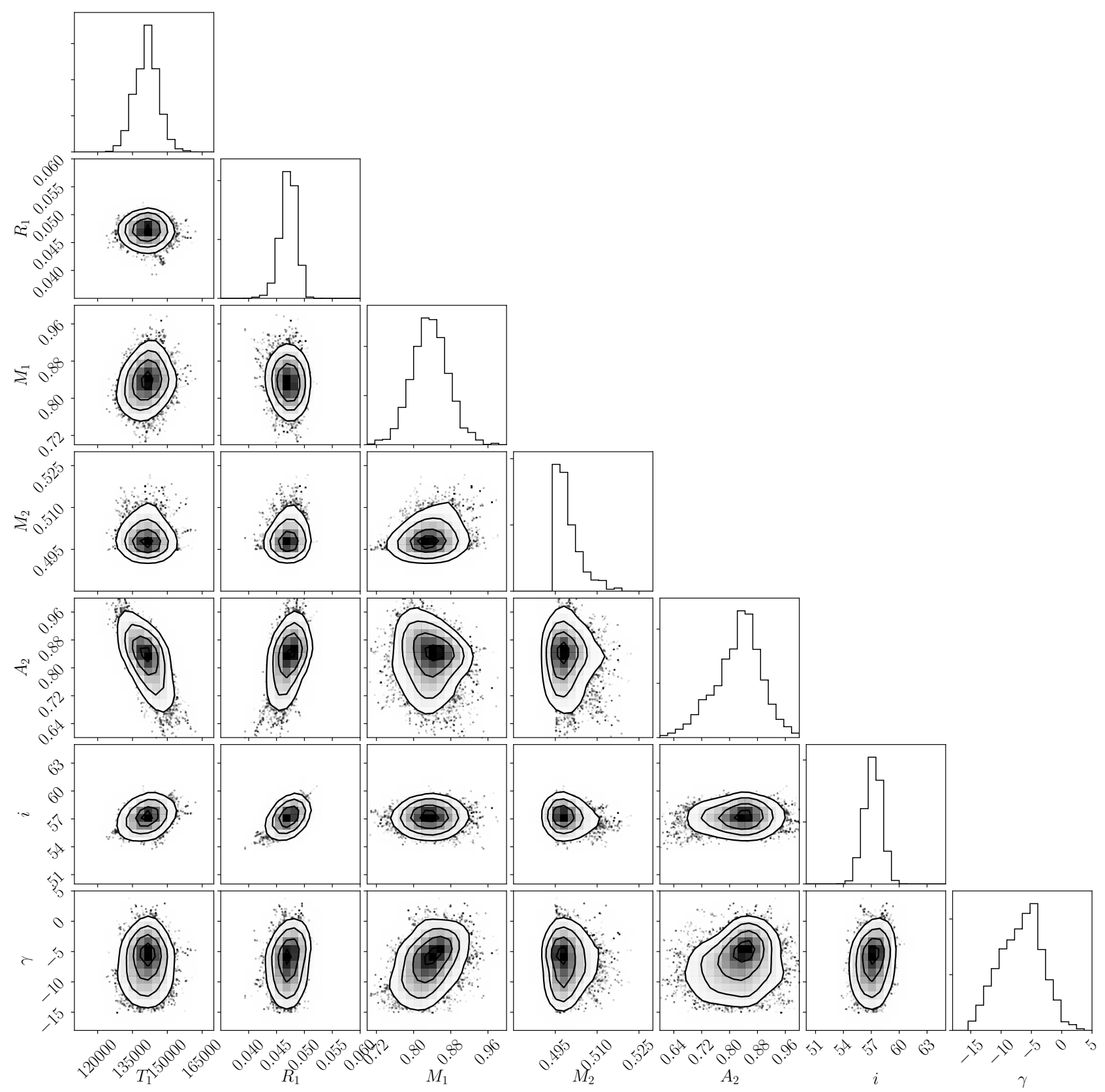

Figure 4. A corner plot (made using CoRnER; Foreman-Mackey 2016) of the PHOEBE2 MCMC posteriors for the primary temperature $\left(\boldsymbol{T}_{1}\right)$, radius $\left(\boldsymbol{R}_{1}\right)$ and mass $\left(M_{1}\right)$, and secondary mass $\left(M_{2}\right)$ and albedo $\left(A_{2}\right)$, as well as the binary orbital inclination $(i)$ and systemic velocity $(\gamma)$.

ETHOS 1 based on three-band photometry and radial velocity measurements of the irradiated emission line complex originating from the secondary. Those authors find fits consistent with the central star being an extremely hot white dwarf with a mass of either 0.7 or $0.78 \mathrm{M}_{\odot}$. However, perhaps due to the lack of observations between phases $0.4-0.7$ where the emission lines are strongest, less than half of their spectra could be used to derive the radial velocity curve (see Figure 3 c.f. their figure 4 ). In deriving the mass function of the binary, they include a K-correction in order to account for the fact that the emission line complex is not reflective of the dynamical (centre-of-mass) velocity of the companion (but rather the centre of light velocity which is skewed towards the first Lagrange point due to the irradiation from the hot primary; Exter et al. 2003; Miszalski et al. 2011). However, for such a large irradiation effect, their correction of only 7-9 $\mathrm{km} \mathrm{s}^{-1}$ seems rather small - for example, applying the equation of Wood et al. (1995), which derives the K-correction assuming that all parts of the irradiated hemisphere contribute equally to the emission while the outward facing hemisphere does not contribute at all, would imply a K-correction of $\sim 15 \mathrm{~km} \mathrm{~s}^{-1}$ for their model parameters. Note that this is likely the minimum correction as not all parts of the irradiated hemisphere will contribute equally, with the most irradiated regions (i.e. those closest to the primary) contributing more. Thus, the potentially underestimated K-correction could, if the orbital inclination of the 
Table 2. Best-fitting model parameters for the central star. Parameters marked with an asterisk were derived using the MTRR of Eker et al. (2018).

\begin{tabular}{|c|c|c|}
\hline Parameter & \multicolumn{2}{|c|}{ Best fitting value } \\
\hline Primary mass $\left(\mathrm{M}_{\odot}\right)$ & 0.84 & \pm 0.04 \\
\hline Primary temperature $(\mathrm{kK})$ & 141 & \pm 6 \\
\hline Primary radius $\left(\mathrm{R}_{\odot}\right)$ & 0.047 & \pm 0.002 \\
\hline Secondary mass $\left(\mathrm{M}_{\odot}\right)$ & 0.50 & \pm 0.01 \\
\hline Secondary temperature $^{\star}(\mathrm{kK})$ & 3.5 & \pm 0.1 \\
\hline Secondary radius ${ }^{\star}\left(\mathrm{R}_{\odot}\right)$ & 0.48 & \pm 0.01 \\
\hline Secondary albedo & 0.83 & $\begin{array}{l}+0.06 \\
-0.08\end{array}$ \\
\hline Binary inclination $\left({ }^{\circ}\right)$ & 57.3 & $\begin{array}{l}+0: 80 \\
-1.0\end{array}$ \\
\hline Systemic velocity, $\gamma\left(\mathrm{km} \mathrm{s}^{-1}\right)$ & -6.5 & $\begin{array}{l}-1.0 \\
+3: 2 \\
-4.0\end{array}$ \\
\hline
\end{tabular}

model holds (which our modelling indicates is likely not the case), lead to a significantly underestimated mass for the primary star (Exter et al. 2003).

It is unclear how Mitrofanova et al. (2016) derive the Kcorrection, other than that it is apparently based on synthetic spectra. However, it clearly does not correspond to the centre-of-light of the irradiated hemisphere (which is the approach taken in PHOEBE2, roughly equivalent to a K-correction of $\sim 25 \mathrm{~km} \mathrm{~s}^{-1}$ for their model parameters). For the K-correction of Mitrofanova et al. (2016) to be valid, the irradiated emission lines would have to originate from the entire surface of the companion (even the non-irradiated hemisphere) or not be produced in the most irradiated regions of the irradiated hemisphere (i.e. restricted to a ring/band perpendicular to the orbital plane and displaced slightly towards the first Lagrange point). Importantly, applying a larger K-correction to the Mitrofanova et al. (2016) models would imply a primary mass approaching the Chandrasekhar limit and thus an exceptionally massive progenitor (our model has a greater inclination and thus avoids this issue).

Mitrofanova et al. (2016) did not consider the expected link between nebular inclination and binary orbital plane (Hillwig et al. 2016) and choose to restrict the inclinations of their models to inclinations between $35-45^{\circ}$, based on the light curve morphology. We do allow our model fits to explore inclinations as low as those found by Mitrofanova et al. (2016), but also extend the range upwards to encompass inclinations compatible with that of the nebula (the final range explored being $35^{\circ}<i<70^{\circ}$ ). Ultimately, we find that our best-fitting model presents with an inclination in good agreement with that of the nebula $\left(57.3^{\circ} \pm 1.0^{\circ}\right.$ c.f. $\left.60^{\circ} \pm 5^{\circ}\right)$, strongly indicating that ETHOS 1 does actually follow the expected correlation between binary plane and nebular symmetry axis just as for every other case where this has been tested (Hillwig et al. 2016). If we force our models to the inclination found by Mitrofanova et al. (2016), the fit becomes appreciably worse (particularly in the $i$-band, in which they did not have data) and the primary mass tends towards the Chandrasekhar limit.

We find that we are able to fit the observed light and radial velocity curves using a model secondary which follows a standard MTRR for main-sequence stars. The model secondaries of Mitrofanova et al. (2016), on the other hand, have much larger radii than predicted using the same MTRR. Although there is a precedence for secondaries with inflated radii in post-CE binary central stars (e.g.; Jones et al. 2015), the radii of the Mitrofanova et al. (2016) model secondaries are very large for their model masses (being more than a factor of 3 and 2 larger than typical for their two model solutions, respectively). However, it is unclear how their secondary masses are constrained in the modelling (the radial velocities of the primary star are not measured). Thus, given that we can model the system with a companion which follows a standard MTRR for mainsequence stars, we conclude that there is no evidence of inflation in the secondary of ETHOS 1 . This is a particularly interesting conclusion given that most post-CE central stars are found to present at least some level of inflation (Jones et al. 2015, 2020a). However, ETHOS 1 would not be the first such case (e.g.; the central star of M 3-1, Jones et al. 2019, although this may be a special case as the secondary is very close to Roche-lobe filling). Moreover, studies of more evolved post-CE white-dwarf-main-sequence binaries find that the M-dwarf companions in those systems are indistinguishable from field systems (Parsons et al. 2018), indicating that such inflation may not be a universal characteristic of the CE or, at the very least, not so long-lived.

Another likely difference in our modelling approach is in the choice of secondary albedo, which was a free parameter in our models (encompassing the physically accepted range of 0.6-1.0). Unfortunately, Mitrofanova et al. (2016) do not quote the secondary albedo used in their modelling, however if it were fixed to the theoretical value for fully-convective stars ( 0.6; Ruciński 1969), then this could mean that a larger primary temperature (as in both their models) or a physically larger companion would be required to replicate the observed photometric amplitudes if the true albedo were higher - just as we find. Similar studies of highly irradiated binaries do tend to empirically derive larger albedos (Rafert \& Twigg 1980), while more-detailed theoretical studies similarly predict larger albedos $(\sim 0.75-0.8$, in line with that derived here $)$ at the effective temperature of their model companion (Claret 2001).

The models of Mitrofanova et al. (2016) imply a distance (in order to match the observed brightness, assuming the extinction determined by Miszalski et al. 2011, from the nebular spectrum) of between 4-6 kpc, while our model would indicate a distance of $\sim 4.2 \mathrm{kpc}$. No distance within this range can be discarded based on the observed kinematical ages of both the central regions and jets, which would be $4-10 \mathrm{kyr}$ depending on the model. The $0.78 \mathrm{M}_{\odot}$ model of Mitrofanova et al. (2016) provides the best match to the distance derived using the $\mathrm{H} \alpha$-surface-brightness-radius relation of Frew et al. (2016) at $6.92 \pm 1.26 \mathrm{kpc}$. However, there is some indication that post-CE PNe are less massive and less luminous than their single-star counterparts (Frew 2008; Corradi et al. 2015), which would lead to an overestimated distance. As such, the implied distances do not rule out any particular model (our own or either model of Mitrofanova et al. 2016).

Mitrofanova et al. (2016) also chose to make their model primaries lie on the evolutionary tracks of Bloecker (1995), which do not take into account the significant advancements in our understanding of AGB star evolution from the last decades (Miller Bertolami 2016). In any case, these models are for single stars and may not necessarily be entirely representative of post-CE systems (Miller Bertolami 2017; Jones et al. 2019, 2020a). Putting these considerations aside, the best fitting $0.7 \mathrm{M}_{\odot}$ model of Mitrofanova et al. (2016) lies on the evolutionary track of Bloecker (1995) for an age of $\sim 700 \mathrm{yr}^{4}$, inconsistent with the larger kinematical ages required for their models to reproduce the observed angular dimensions of ETHOS 1.

Based on the initial-to-final-mass relation of Cummings et al.

4 No Bloecker (1995) tracks are available for a remnant mass of $0.78 \mathrm{M}_{\odot}$, so it is not possible to test the age of the solution of Mitrofanova et al. (2016) with this mass. However, interpolation of the higher mass tracks at $0.84 \mathrm{M}_{\odot}$ and lower mass at $0.7 \mathrm{M}_{\odot}$ indicate that this solution would have a similarly young post-AGB age. 
(2018), the initial mass of the CSPN would have been 2.7-3.3 $\mathrm{M}_{\odot}$ for the remnant masses derived by Mitrofanova et al. (2016), and $\sim 3.5 \mathrm{M}_{\odot}$ for the remnant in our model. All of which are rather massive for the Galactic latitude and estimated distance of ETHOS 1. Single-star nucleosynthesis models (e.g.; Ventura et al. 2013; Karakas \& Lugaro 2016) predict that third dredge-up should be activated in stars with initial masses in these ranges, ultimately leading to elevated $\mathrm{C}$ and s-process element abundances in the surrounding PN. One may thus look for indicators of the initial mass in the nebular abundances of ETHOS 1. Unfortunately, the intrinsic faintness and density-bounded nature of the nebula limits the drawing of strong conclusions, with no s-process lines detected and the $\mathrm{C} / \mathrm{O}$ ratio typically measured using faint lines in the UV (outside the range of the available observations).

As highlighted in García-Rojas et al. (2018), the He/H ratio can also be a probe of the initial mass - particularly for more massive progenitors when combined with the N/O ratio. In the central nebula of ETHOS 1 , the $\mathrm{He}$ II $\lambda 4686$ line is brighter than $\mathrm{H} \beta$, however no $\mathrm{He}$ I emission was detected. Note that $\mathrm{He}$ I $\lambda 4471$ was identified in the SE jet (although only weakly), but the lines fluxes of the jets are difficult to interpret in terms of chemical abundances due to their shock-excited nature, thus we do not consider them here. Assuming that all $\mathrm{He}$ in the central nebula is in the form of $\mathrm{He}^{++}$, the obtained abundance is $12+\log (\mathrm{He} / \mathrm{H})=11.01 \pm 0.04$ - relatively low and perhaps indicative of a low initial mass. However, there are significant degeneracies between models, depending on the physics assumed and the metallicity (Ventura et al. 2013; Karakas \& Lugaro 2016), meaning that initial masses up to $\sim 4.5 \mathrm{M}_{\odot}$ could fit with such an abundance. As such, the nebular abundances (based on the few nebular lines observed by Miszalski et al. 2011), cannot be used to distinguish effectively between our model and those of Mitrofanova et al. (2016).

As already mentioned, the $\mathrm{He}$ II $\lambda 4686$ line is brighter than $\mathrm{H} \beta$ in ETHOS 1 - a clear sign that the nebula is density-bounded (i.e. all the nebular material has been ionised). However, the ionised mass of the nebula is found to be strikingly low at roughly $0.002 \times D^{2} \mathrm{M}_{\odot}$, where $D$ is the distance to ETHOS 1 in kpc (Santander-García et al. 2019, Santander-García et al., in prep). Similarly minuscule masses have been noted for several other post-CE PNe (Corradi et al. 2015), seemingly at odds with the idea that the nebula represents the majority of the progenitor's envelope (which would be at least an order of magnitude more massive) rapidly ejected during the $\mathrm{CE}$ event (Corradi et al. 2014, 2015).

\section{CONCLUSIONS}

We have presented a detailed study of the binary nucleus of ETHOS 1, indicating that the central star is a $\sim 140 \mathrm{kK}$ pre-whitedwarf of mass $\sim 0.84 \mathrm{M}_{\odot}-$ making it one of the hottest and mostmassive post-CE central stars known (see e.g.; the central star of NGC 2392; Miszalski et al. 2019). A similar study by Mitrofanova et al. (2016) found model fits with central star masses of 0.7 and $0.78 \mathrm{M}_{\odot}$, in relatively good agreement with the mass derived here in spite of finding a significantly lower orbital inclination (the differences between their analyses and the one presented here are discussed at length in Section 3.1).

The orbital inclination of our model is found to be in good agreement with the nebular inclination as derived via morphokinematical modelling Miszalski et al. (2011). This is at odds with the findings of Mitrofanova et al. (2016), who found a binary inclination discrepant by roughly $20^{\circ}$ - which would make ETHOS 1 the first post-CE PN not to follow the expected correlation between binary plane and nebular symmetry axis (Hillwig et al. 2016). It should also be noted that, assuming the measured radial velocities are representative of the companion's centre of light, the mass of the primary would be much larger (approaching the Chandrasekhar limit) for the orbital inclination found by Mitrofanova et al. (2016).

Although the radius and mass of the companion are relatively poorly constrained in the PHOEBE2 modelling (due to the binary's non-eclipsing nature and the lack of RV measurements from the primary), a good fit was found using parameters compatible with being a normal main-sequence star. This could be an indication that, in the case of ETHOS 1, the mass transfer which led to the formation of the jets did not significantly impact upon the physical parameters of the companion (unlike in the majority of well-studied, main-sequence companions to post-CE PN central stars; Jones et al. 2015).

To conclude, our modelling indicates that the central star of ETHOS 1 is one of the hottest (and most massive) known inside a post-CE PN. As the system is not eclipsing, nor double-lined, the parameters of the secondary are difficult to constrain independently. However, models using a MTRR find satisfactory solutions indicating that its properties are comparable to typical early M-type stars. The binary inclination is consistent with lying perpendicular to the nebular symmetry axis, as determined by spatio-kinematical modelling, adding further weight to the empirical correlation found by Hillwig et al. (2016). Indeed, adding ETHOS 1 to the eight systems in Hillwig et al. (2016), the likelihood of chance alignment drops to less than $0.00002 \%$.

\section{ACKNOWLEDGEMENTS}

The authors would like to thank the referee, Prof. Todd Hillwig, for his extremely insightful report which greatly improved the paper. The authors are also grateful to Prof. Bruce Balick for helping to obtain the APO data, and to Prof. Z. Eker for providing the corrected form of the mass-radius relationship.

JM acknowledges the support of the ERASMUS+ programme in the form of a traineeship grant. DJ, JGR, PRG and RLMC acknowledge support from the State Research Agency (AEI) of the Spanish Ministry of Science, Innovation and Universities (MCIU) and the European Regional Development Fund (FEDER) under grant AYA2017-83383-P. DJ and JGR also acknowledge support under grant P/308614 financed by funds transferred from the Spanish Ministry of Science, Innovation and Universities, charged to the General State Budgets and with funds transferred from the General Budgets of the Autonomous Community of the Canary Islands by the Ministry of Economy, Industry, Trade and Knowledge. MSG acknowledges support from the Spanish Ministry of Science, Innovation, and Universities under grant AYA2016-78994-P. PS thanks the Polish National Center for Science $(\mathrm{NCN})$ for support through grant 2015/18/A/ST9/00578.

The authors thankfully acknowledge the technical expertise and assistance provided by the Spanish Supercomputing Network (Red Española de Supercomputación), as well as the computer resources used: the LaPalma Supercomputer, located at the Instituto de Astrofísica de Canarias.

Based on observations made with the Isaac Newton Telescope and the William Herschel telescope both operated by the Isaac Newton Group of Telescopes, with the Mercator Telescope operated by the Flemish community, and with the Liverpool Telescope operated by by Liverpool John Moores University with financial support from 
the UK Science and Technology Facilities Council, all of which reside on the island of La Palma at the Spanish Observatorio del Roque de los Muchachos of the Instituto de Astrofísica de Canarias. This research made use of Astropy, ${ }^{5}$ a community-developed core Python package for Astronomy (Astropy Collaboration et al. 2013, 2018).

\section{DATA AVAILABILITY}

All raw LT-IO:O, INT-WFC and WHT-ISIS data are available from the respective online archives, while the Mercator-Merope and ARC-DIS data are available upon reasonable request from the authors. All extracted photometry and radial velocities are available in the article or from VizieR at the CDS.

\section{References}

Astropy Collaboration et al., 2013, A\&A, 558, A33

Astropy Collaboration et al., 2018, AJ, 156, 123

Barbary K., 2016, The Journal of Open Source Software, 1, 58

Bertin E., Arnouts S., 1996, A\&AS, 117, 393

Bloecker T., 1995, A\&A, 299, 755

Boffin H. M. J., Jones D., 2019, The Importance of Binaries in the Formation and Evolution of Planetary Nebulae. Springer, doi:10.1007/978-3-03025059-1

Boffin H. M. J., Miszalski B., Rauch T., Jones D., Corradi R. L. M., Napiwotzki R., Day-Jones A. C., Köppen J., 2012, Science, 338, 773

Castelli F., Kurucz R. L., 2003, in Piskunov N., Weiss W. W., Gray D. F., eds, IAU Symposium Vol. 210, Modelling of Stellar Atmospheres. p. A20 (arXiv: astro-ph/0405087)

Claret A., 2001, MNRAS, 327, 989

Claret A., Cukanovaite E., Burdge K., Tremblay P. E., Parsons S., Marsh T. R., 2020, A\&A, 634, A93

Corradi R. L. M., et al., 2011, MNRAS, 410, 1349

Corradi R. L. M., et al., 2014, MNRAS, 441, 2799

Corradi R. L. M., García-Rojas J., Jones D., Rodríguez-Gil P., 2015, ApJ, 803,99

Craig M., et al., 2017, astropy/ccdproc: v1.3.0.post1, doi:10.5281/zenodo.1069648, https://doi.org/10.5281/ zenodo. 1069648

Cummings J. D., Kalirai J. S., Tremblay P. E., Ramirez-Ruiz E., Choi J., 2018, ApJ, 866, 21

Currie M. J., Draper P. W., Berry D. S., Jenness T., Cavanagh B., Economou F., 2008, in R. W. Argyle, P. S. Bunclark, \& J. R. Lewis ed., Astronomical Society of the Pacific Conference Series Vol. 394, Astronomical Data Analysis Software and Systems XVII. pp 650-+

Davignon G., et al., 2004, in Moorwood A. F. M., Iye M., eds, Society of Photo-Optical Instrumentation Engineers (SPIE) Conference Series Vol. 5492, SPIE. pp 871-879, doi:10.1117/12.550773

De Marco O., Long J., Jacoby G. H., Hillwig T., Kronberger M., Howell S. B., Reindl N., Margheim S., 2015, MNRAS, 448, 3587

Dhillon V. S., Privett G. J., Duffey K. P., 2001, Starlink User Note 167.6. Rutherford Appleton Laboratory

Douchin D., et al., 2015, MNRAS, 448, 3132

Eker Z., et al., 2018, MNRAS, 479, 5491

Exter K. M., Pollacco D. L., Bell S. A., 2003, MNRAS, 341, 1349

Foreman-Mackey D., 2016, The Journal of Open Source Software, 1, 24

Frew D. J., 2008, PhD thesis, Department of Physics, Macquarie University, NSW 2109, Australia

Frew D. J., Parker Q. A., Bojičić I. S., 2016, MNRAS, 455, 1459

García-Rojas J., Delgado-Inglada G., García-Hernández D. A., Dell'Agli F., Lugaro M., Karakas A. I., Rodríguez M., 2018, MNRAS, 473, 4476
Hillwig T. C., Jones D., De Marco O., Bond H. E., Margheim S., Frew D., 2016, ApJ, 832, 125

Hillwig T. C., Frew D. J., Reindl N., Rotter H., Webb A., Margheim S., 2017, AJ, 153, 24

Horne K., 1986, PASP, 98, 609

Horvat M., Conroy K. E., Pablo H., Hambleton K. M., Kochoska A., Giammarco J., Prša A., 2018, ApJS, 237, 26

Horvat M., Conroy K. E., Jones D., Prša A., 2019, ApJS, 240, 36

Husser T. O., Wende-von Berg S., Dreizler S., Homeier D., Reiners A., Barman T., Hauschildt P. H., 2013, A\&A, 553, A6

Jones D., Boffin H. M. J., 2017, Nature Astronomy, 1, 0117

Jones D., Boffin H. M. J., Rodríguez-Gil P., Wesson R., Corradi R. L. M., Miszalski B., Mohamed S., 2015, A\&A, 580, A19

Jones D., Boffin H. M. J., Sowicka P., Miszalski B., Rodríguez-Gil P., Santand er-García M., Corradi R. L. M., 2019, MNRAS, 482, L75

Jones D., et al., 2020a, A\&A, p. arXiv:2007.08960

Jones D., et al., 2020b, ApJS, 247, 63

Karakas A. I., Lugaro M., 2016, ApJ, 825, 26

Miller Bertolami M. M., 2016, A\&A, 588, A25

Miller Bertolami M. M., 2017, in Liu X., Stanghellini L., Karakas A., eds, IAU Symposium Vol. 323, Planetary Nebulae: Multi-Wavelength Probes of Stellar and Galactic Evolution. pp 179-183 (arXiv: 1611.09801), doi:10.1017/S1743921317001533

Miszalski B., Acker A., Moffat A. F. J., Parker Q. A., Udalski A., 2009, A\&A, 496, 813

Miszalski B., Corradi R. L. M., Boffin H. M. J., Jones D., Sabin L., Santander-García M., Rodríguez-Gil P., Rubio-Díez M. M., 2011, MNRAS, 413, 1264

Miszalski B., Boffin H. M. J., Corradi R. L. M., 2013, MNRAS, 428, L39

Miszalski B., Manick R., Van Winckel H., Escorza A., 2019, PASA, 36, e018

Mitchell D. L., Pollacco D., O’Brien T. J., Bryce M., López J. A., Meaburn J., Vaytet N. M. H., 2007, MNRAS, 374, 1404

Mitrofanova A. A., Shimansky V. V., Borisov N. V., Spiridonova O. I., Gabdeev M. M., 2016, Astronomy Reports, 60, 252

Parsons S. G., et al., 2018, MNRAS, 481, 1083

Prša A., et al., 2016, ApJS, 227, 29

Rafert J. B., Twigg L. W., 1980, MNRAS, 193, 79

Ruciński S. M., 1969, Acta Astron., 19, 245

Santander-García M., Jones D., Alcolea J., Wesson R., Bujarrabal V., 2019, in Highlights on Spanish Astrophysics X. pp 392-396 (arXiv: 1810.09296)

Shortridge K., et al., 2004, Starlink User Note 86.21. Rutherford Appleton Laboratory

Steele I. A., et al., 2004, in Oschmann Jacobus M. J., ed., Society of PhotoOptical Instrumentation Engineers (SPIE) Conference Series Vol. 5489, SPIE. pp 679-692, doi:10.1117/12.551456

Ventura P., Di Criscienzo M., Carini R., D’Antona F., 2013, MNRAS, 431, 3642

Wood J. H., Robinson E. L., Zhang E. H., 1995, MNRAS, 277, 87

This paper has been typeset from a $\mathrm{T}_{\mathrm{E}} \mathrm{X} / \mathrm{L} \mathrm{AT} \mathrm{E} \mathrm{X}$ file prepared by the author.

5 http://www.astropy.org 